\title{
Conference on Gene Therapy, Royal College of Physicians, London, 1 March 1990
}

The logic of correcting or replacing the faulty gene responsible for a genetic disorder with the normal or wild type gene has been obvious for some time, as this plaintive poem from Cuthbert Duke's Hunterian lecture in 1952 shows.

\footnotetext{
"In my youth," Father William replied with grin,

"I was told that a gene had mutated,

That all who carried this dominant gene

To polyps and cancer were fated.
}

"It seemed rather bad luck-I was then but nineteen

So I went and consulted a quack,

Who took a firm grip on my dominant gene

And promptly mutated it back."

(This was quoted by Professor Sikora in his masterly Watson Smith lecture on the molecules of cancer-a fitting finale to a stimulating conference.) However, the technology for achieving such a feat has only recently potentially become attainable. The conference on Gene Therapy at the Royal College of Physicians on 1.3.90 provided fascinating insights into progress in this area.

Professor Weatherall opened the conference with a very clear overview of the realistic prospects for gene therapy. He outlined five basic conditions for success.

(1) The genotype and the variability of the relevant phenotype must be thoroughly understood, including factors which alter the phenotypic effect of the mutant gene, such as heterogeneity of mutations, modification by other loci, contiguous gene syndromes, and epigenetic factors (including $\mathrm{X}$ inactivation, cytoplasmic inheritance, and parental imprinting).

(2) The gene must be isolated with its regulatory genes. The importance of understanding the mechanisms of gene regulation was stressed. A housekeeping gene', which is expressed at a low level in most tissues with relatively little fine alteration of expression, will have a different type of gene regulation from that of tissue or developmentally specific genes, which are expressed in limited cell populations, possibly only at certain stages of development, and regulated by tightly controlled mechanisms. Regions of the gene involved in regulation include standard $5^{\prime}$ gene promoters and $3^{\prime}$ terminator regions acting in cis (close to the gene, on the same chromosome), although trans acting regions (elsewhere in the genome) may also be important, and also DNA binding proteins.

(3) The target cell for gene therapy must be defined and accessible. Germline therapy is not currently considered for use in man but somatic cell targeting is a realistic option. The most appropriate cells are haemopoietic cells, fibroblasts, endothelial cells, hepatocytes, and keratinocytes, as they are relatively easily accessible and grow rapidly, and in the case of bone marrow, early undifferentiated multipotent cells are available. Transformed stem cells could pass on the genetic change to a large (more differentiated) cell population, this overcoming the need for frequent reinfection.

(4) An efficient and stable transfer vector is important. The means of transfecting target cells were outlined; physical methods such as direct DNA insertion are relatively inefficient, but the use of retrovirus vectors and recombinational methods are more feasible. Potential difficulties of stem cell gene transfection were outlined: only a small proportion of marrow cells are stem cells and the control of their differentiation is poorly understood.

(5) Proliferative advantage and long survival of the treated cell population is required. Transfection may not confer a proliferative advantage to such cells that do transfect, and knowledge of factors influencing their survival and the time taken to differentiate is important. High levels of expression of these cells is essential so that gene regulation is critical.

Professor Weatherall considers that the ethical issues concerning somatic gene therapy are no different from those of other therapeutic methods, and that the pros and cons of gene therapy should be evaluated fully against the benefits of existing therapies. Initially, new and potentially unsafe treatments should only be applied to genetic diseases which are life threatening and not amenable to conventional therapy. Normal standards of therapeutic safety would apply. Ethical issues regarding 
germline therapy are somewhat different, as this may be more difficult to reverse and would affect future generations.

Specific issues were taken up by subsequent speakers in considerable detail. Professor Mulligan, from the Massachusetts Institute of Technology, described retroviral vectors and explained that the viral protein coding sequences can be replaced by the therapeutic gene in a provirus which would then be packaged, when combined with helper viral particles, to form a producer cell line that makes only the altered viral particles. The expression of such particles is dependent on cell replication. The viral receptor specifies the cell type which the virus will enter. $\mathrm{He}$ suggested that the diseases most suitable for treatment with gene therapy include blood dyscrasias, such as thalassaemia and sickle cell disease, diseases of lymphocyte function, such as $\mathrm{X}$ linked and Swiss type SCID and ADA deficiency, lysosomal storage diseases, and familial hypercholesterolaemia. It is uncertain whether gene function can be maintained regardless of position in the genome, and whether gene expression will be stable and appropriate. The possibility of an immune response to the new gene product is an important practical issue. In a mouse model of ADA deficiency, injection of marrow cells transfected with the normal gene can result in persistent expression of ADA, but a high level of initial transfection is needed. In the Watanabe rabbit, LDL receptor genes can be transfected into hepatocytes which, when introduced into the portal circulation of hypercholesterolaemic animals, results in LDL receptor expression, but so far this effect has only been very short lived (up to two weeks).

Professor Green from the Harvard Medical School discussed the use of keratinocytes as target cells for gene therapy. These cells are easily cultured and can form an epithelial cell layer in vitro after transfection. This can be detached as a whole and transplanted to a recipient as a long lasting graft. Such a culture can expand by a factor of 5000 in three weeks, and transfection with genes encoding diffusible proteins, such as factor IX or human growth hormone, results in production of these proteins in vitro, but this is often a disappointingly short lived effect, lasting less than two weeks. One advantage of successful gene therapy using this system would be that if things went wrong the graft could be removed. However, in skin disorders where the defective gene is non-diffusible, a total body skin graft would be required for successful treatment of the genetic disorder!

Professor Brownlee from Oxford discussed the possibility of using gene therapy for the treatment of haemophilia B. Post-translational modification of the normal gene product is required for normal factor IX clotting activity, and for this the transfected gene product must be appropriately modified by carboxylation in the recipient. Initial experiments in nude mice showed the successful production of factor IX in deficient mice treated with fibroblasts transfected with the normal factor IX gene, but this was a disappointingly short lived effect (lasting for one week only), and at a level of activity of $20 \%$ of normal. It was suggested that using a longer lived cell type and enhancing post-translational carboxylation could improve these results.

Dr K Thomas from the University of Utah discussed the technique of gene targeting, using cultured cells from mouse preimplantation blastocysts. Successfully targeted cells could be transferred into a blastocyst in a host mouse and the effects of the gene therapy studied in the resulting offspring, with the possibility of germline gene expression in some cases. The advantage of gene targeting over the technique of insertion of a normal gene in addition to the mutant gene is that the position of the targeted gene is the natural position of the wild type gene, and therefore more likely to be correctly regulated, and the theoretical problem of recombination between an additional normal gene and the mutant gene, when still present, is avoided. The gene targeting process requires the synthesis of a linear vector containing the normal gene with flanking regions of homology with the mutant gene, this allowing natural recombination to occur, with the result that the normal gene replaces the mutant allele in some cells. Such a method is critically dependent upon a reasonably high rate of transformation, and a system which enables trans formed genes to be selected for in vitro. Methods of selection include the inclusion in the vector of a neomycin sensitivity gene and the HPRT gene, which can be selected for and against by growing the cells in specific media. The neomycin sensitivity gene can then be spliced out at a later stage. Using this system of 'dominant selection' for transformed genes, and especially where large regions of homology are present near the target gene (which increases the rate of recombination), an efficiency of transformation of considerably greater than 1 in 1000 cells can be achieved. There was no evidence for random insertion of transfected DNA.

Dr Melton (from Edinburgh University) developed the discussion of gene targeting and its use in the study of the control of gene expression. Using promoter regions with the transfecting HPRT gene vector in studies in HPRT deficient mice, he showed that gene expression could be altered by the use of different promoters. Using transfection with the normal HPRT gene and variable amounts of promoter sequences, the optimal conditions for HPRT expression in recipient mice can be established, and the relative expression of HPRT in different tissues studied to ensure that this is appropriate. The results so far are encouraging in terms of reasonably normal tissue specific regulation of the gene in recipient mice.

Dr Grosveld from the National Institute for Medical 
Research further discussed gene regulation and its relevance to gene therapy, using the $\beta$ globin gene model. In an elegant series of experiments in mice, where the expression of the $\beta$ globin gene was studied after transfection with genes containing variable stretches of 5' promoter DNA sequences, he was able to show the presence of a dominant control region which controls the expression of the $\beta$ globin gene with an effect proportional to the number of copies of the controlling gene but independent of its position. Further studies showed that gene order was important as there appeared to be competition between different contiguous genes for regulation, dependent upon their positions relative to each other. In addition, genes have expression patterns altered by developmental stage, embryonic and adult genes responding differently to the same promoter regions. An interesting observation in this study was that certain introns were necessary for the expression of the $\beta$ globin genes and for termination of RNA translation. A mouse model has been produced with the sickle cell disease genotype and phenotype, and this can be used for the study of the optimal degree of expression of therapeutically inserted $\beta$ globin genes.

This was a fascinating conference, providing many insights into gene expression and function, and a glimpse into the future for possible gene therapy. At present, however, the many obvious practical and theoretical difficulties of such therapy indicate that we have many years of work ahead before such therapy is widely available.

SHIRLEY HODGSON Division of Medical and Molecular Genetics, Guy's Hospital, Guy's Tower, 7th-8th Floor, London SE1 9RT. 